\title{
SOMATIC SELECTION IN PERENNIAL RYEGRASS
}

\author{
E. L. BREESE, M. D. HAYWARD and A. C. THOMAS \\ Welsh Plont Breeding Stotion, Aberystwyth
}

Received 21.i.65

\section{INTRODUCTION}

MANY permanent grasslands in this country are dense swards managed in such a way as to limit severely the production of seed heads. Under these conditions the frequency of regeneration by sexual means must be extremely low since establishment from any occasional seed set would be largely precluded by the high plant density. It would seem therefore that after an initial establishment phase, nuclear or genotypic differences must play a diminishing role in the advance to, and the maintenance of, sward equilibrium.

The characteristic grass species are termed perennial but in fact their survival is ensured through continuous asexual reproduction. Thus Langer (I956) has developed the concept of the perennial grass plant as a dynamic aggregate of self-reproducing, short-lived plantlets or tillers, whose individual life histories are dependent on season and management.

A recent review by Jinks ( 1964 ) outlines the accumulated evidence for, and the importance of, extranuclear changes in the differentiation and degeneration of asexually reproducing organisms. The question is thus raised as to the extent to which extranuclear changes may mediate modification and adaptation of grass plants to particular managements or ecological niches during successive tiller generations. As a first step it was decided to investigate whether the expression of a particular character could be altered by somatic selection.

\section{THE EXPERIMENT}

Work was carried out on clones from three populations of Lolium perenne. The histories of the populations are of interest in that they represent a gradient in the intensity of selection for persistency. Population 3 derives from an old-established pasture with no record of reseeding, population 17 from a pasture reseeded some 20 years previous to being sampled in $195^{8}$ and population 6738 represents the characteristically non-persistent commercial variety Irish perennial ryegrass.

Populations 3 and 17 are based on a sample of forty genotypes sampled in $195^{8}$ and since maintained by vegetative propagation. They have also been made to yield one seed generation by a mass pollination of the 40 genotypes under isolation. In these populations, therefore, two kinds of clonal material could be utilised; that having complete vegetative continuity with the original population, and so 
having a long history of uninterrupted asexual reproduction (termed original or $c$-clones); and that raised from seed after a single sexual cycle (termed ex-seedling or s-clones). Only seedling material was available in population 6738 .

In the experiment two genotypically distinct $s$-clones were raised from seedlings in each of the three populations, and in populations 3 and 17 two genotypically distinct $c$-clones were initiated by single tillers taken from the vegetatively maintained material. Here, and throughout the course of the experiment, the material was grown in boxes containing a modified John Innes compost. The boxes were kept in a glasshouse supplied with supplementary heat and light during the winter months, which served the double purpose of maintaining vegetative growth and avoiding floral induction. The experiment ran from October 1962 to December 1963 , after an establishment period of 3 months.

The character on which selection was based was rate of tillering which in effect is the rate of asexual reproduction. When all clones were fully established they were broken down into tiller units and three uniform tillers were used to propagate three ramets in each clone. These ramets were allowed to grow until at least twenty new tillers had been formed when the ramet with the highest and the ramet with the lowest tiller-number in each clone were selected as the basis of high and low lines. Selected ramets were again broken down into tiller units and three uniform tillers used to propagate a further 3 ramets of the next selection cycle. Selection was repeated, after suitable intervals, by tiller-propagation from the ramet with the highest tiller number in the high line and from the ramet with the lowest tiller number in the low line.

Four selection cycles (S. I-4) were achieved over I5 months, the time interval between each varying with the season. At each stage of propagation care was taken to avoid obvious differentiation, due to age or other effects, by selecting healthy tillers at the $4^{\text {th-leaf }}$ stage of development. This limited the number of tillers offered by a given ramet and it was found convenient to restrict multiplication to three ramets at any one stage. This of course drastically limited the selection pressure.

It must be emphasised that no measurement of the actual number of tiller generations was obtained during a selection cycle as distinct from the overall increase in tiller-number. At the completion of any cycle selected tillers could have been primary, secondary or of a higher regeneration order with respect to the initiating tiller, provided they were similar in growth stage. Selection cycles must not therefore be confused with tiller generations.

At the end of S.3, in addition to generating S.4, five tillers were taken at random from all three ramets in each selection line in order to assess more critically differences between lines. These were planted in boxes in September so that each family of five daughter tillers 


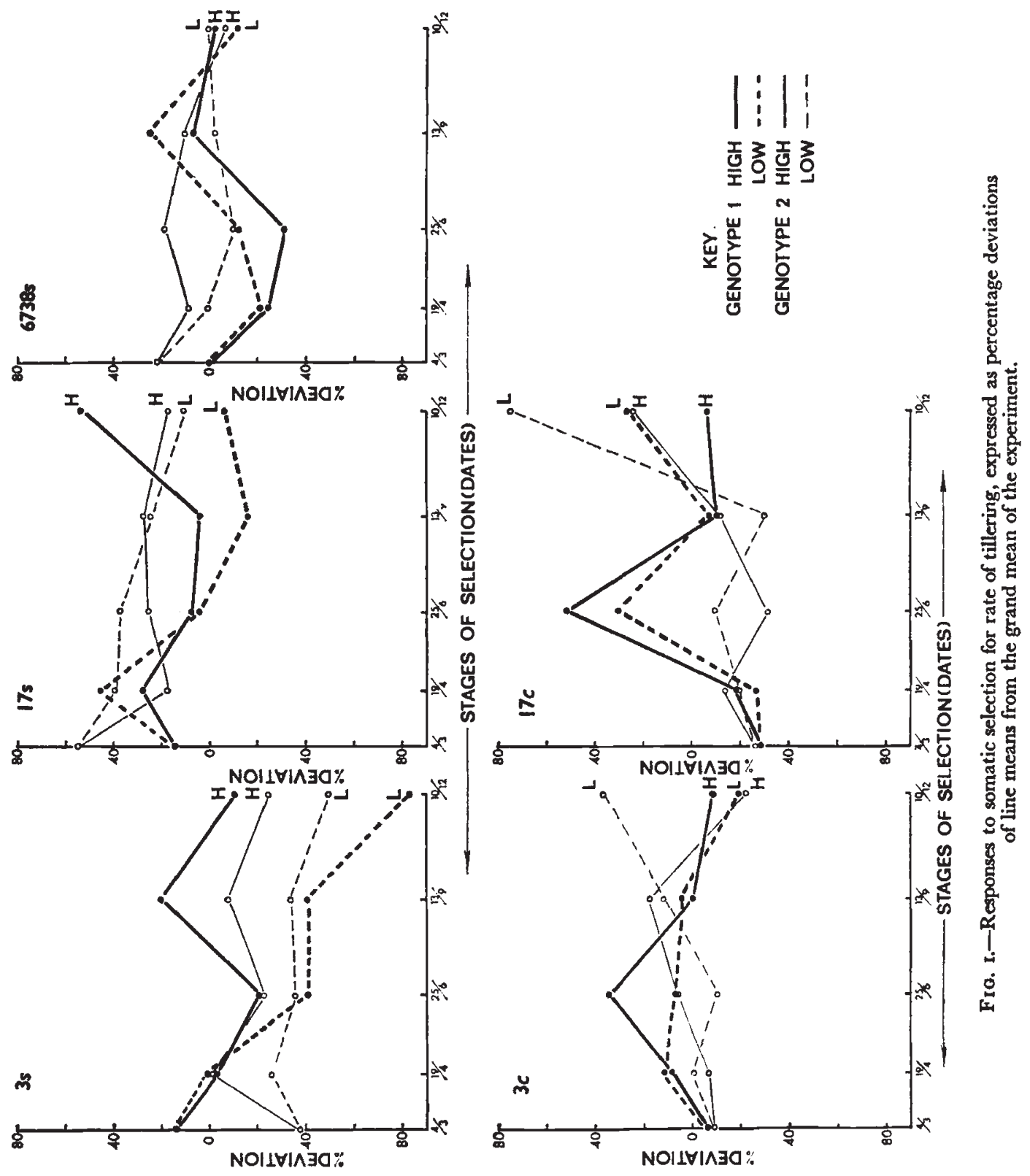


formed a row, and the three family-rows representing a selection line occupied a box. Boxes were randomised in a heated glasshouse receiving supplementary light and the tiller numbers counted in November. This is referred to as the assessment phase.

\section{RESULTS}

The selection cycles

Fig. I shows responses over four cycles of selection for all ten clones. In the absence of suitable controls the response is portrayed as a percentage deviation from the grand mean of all the selection lines

TABLE I

Analysis of variance of differences between high and low selection lines

Data obtained from final tiller-numbers of the 4 selection cycles.

(Totals of 3 ramets per line.)

\begin{tabular}{|c|c|c|c|c|}
\hline \multirow{2}{*}{ Source } & \multicolumn{2}{|c|}{ Seedling-derived $(s)$ clones } & \multicolumn{2}{|c|}{ Original $(c)$ clones } \\
\hline & D.F. & M.S. & D.F. & M.S. \\
\hline $\begin{array}{l}\text { Overall selection } \\
\text { Selection } \times \text { stage } \\
\text { Selection } \times \text { populations } \\
\text { Selection } \times \text { genotypes } \\
\begin{array}{l}\text { populations } \\
\text { Remainder (Error) }\end{array}\end{array}$ & $\begin{array}{r}1 \\
3 \\
2 \\
3 \\
15\end{array}$ & $\begin{array}{l}2700 * * \\
599 \\
1501 * \\
663 \\
286\end{array}$ & $\begin{array}{r}1 \\
3 \\
1 \\
2 \\
12\end{array}$ & $\begin{array}{r}81 \\
816 \\
88 \\
215 \\
269\end{array}$ \\
\hline
\end{tabular}

** $\mathrm{P}<0.01 \quad * 0.01<\mathrm{P}<0.05$

at the appropriate stage of selection. It is thereby possible to compare the magnitude of response with genotypic differences. Deviations are plotted on a time scale.

Response is apparent among the seedling-derived ( $s$ ) clones, more specifically in populations 3 and 17 , but not in the original $(c)$ clones. From these data our criterion as to the reality of effects must come from the consistency of response over selection cycles. A rough but valid measurement of this is provided by the analysis of variance in table I which employs as raw data the differences between tiller counts of selection lines within a clone at the end of each selection cycle (= stage). Data from $s$-clones and $c$-clones are analysed separately. The second order interaction of selection, stage and genotypes, is taken as the basic error of the analysis. The levels of significance shown are calculated assuming a normal distribution; but, in fact, since only deviation in the direction of selection is accepted, the distribution could be regarded as having a single tail and these probability values could be halved. The confidence limits accepted may therefore be regarded as minimal. 


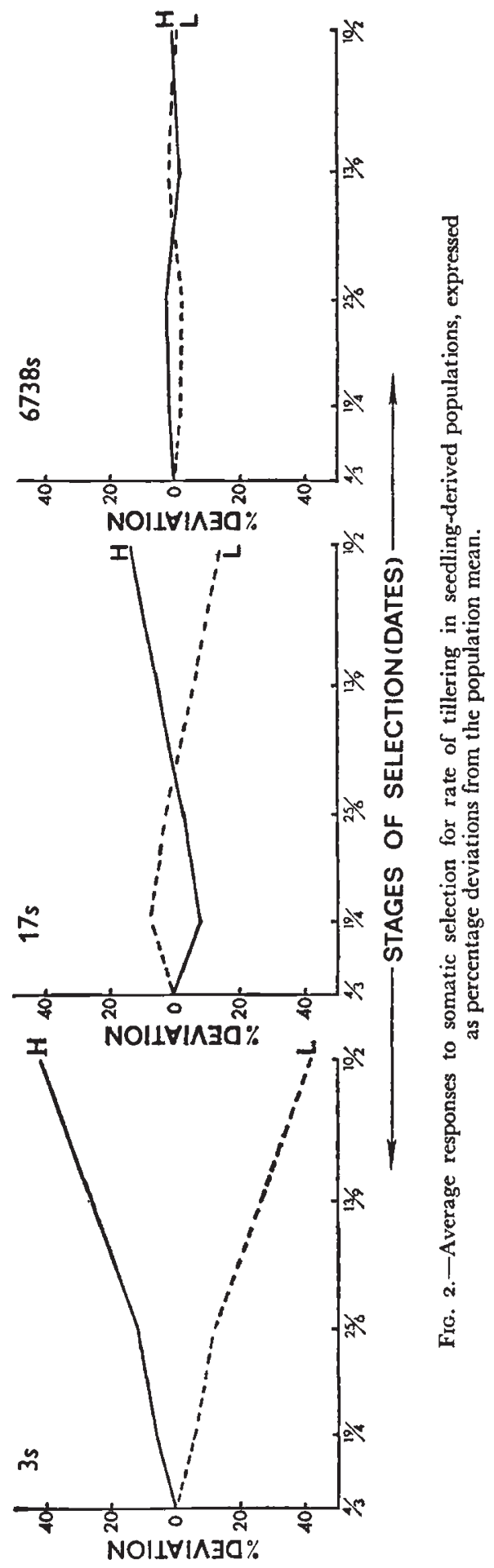


Taking first the s-clones, we find that the item measuring the average response to selection over all clones is significant at the I per cent. level. There is, however, a significant interaction between genotypes and response which indicates a genotypic influence. In the table this interaction item has been broken down to show the effect of populations and of genotypes within populations separately. Although these two items are not significantly different from each other, the former is sufficiently large to suggest that the origin of the genotypes might be important in determining response. Accordingly the average response of genotypes within a population is portrayed in fig. 2. In this instance the response is plotted as the per cent. deviation from the population mean and must therefore appear symmetrical about a mean point.

The apparent influence of the origin of genotypes on the response to selection now becomes clear. Thus s-clones from population 3, which has a long history of persistency under sward conditions, are susceptible to selection: those from population 17 , which has a shorter history of persistency, are less so; while those from population $673^{8}$ show no response to the low selection pressure which this experiment imposed. It will be recalled that 6738 represents a commercial variety where the main emphasis will have been on seed production rather than on continued asexual propagation. Thus the ease of response to somatic selection seems to depend to some extent on a pre-sexual phase of adaptation to asexual propagation.

Turning to the $c$-clones which, in contrast to the s-clones, have not had their long history of asexual propagation interrupted by a sexual cycle, the analyses of variance in table I can provide no evidence of response to selection. In this experiment, therefore, somatic malleability is more a feature of the immediate post-sexual phase.

\section{The ossessment phase}

Mean tiller counts for the assessment phase following S.3 are given in table 2. In that all ramets in the S.3 lines were sampled, this represents a cycle of relaxed selection. The analysis of variance (table 2) again deals with s-clones and $c$-clones separately. Certain comparisons between mean squares have to be effected with some caution because of inadequacies in experimental layout which were accepted for the sake of ease in handling the material. Thus differences between ramet families, which reflect variation between S.3 (parent) ramets, is confounded with positional differences in the box and comparisons between this item and that for ramets within families, which reflects segregational differences within the parent ramets, must be interpreted with caution. The same limitations apply to a comparison of differences between ramet families within lines and differences in the upper hierarchies of the experiment, since the latter have box differences confounded. But here the limitation is less serious since only direct responses are accepted and box differences 
could equally serve to diminish or enhance these effects. Hence the difference between families is taken as the basic error of the experiment. The levels of significance shown are calculated in all cases

TABLE 2

Tiller-numbers after 3 cycles of somatic selection

Lower values are means of 3 parent ramets grown 26th June to 12 th September. Upper values are means of 15 daughter ramets grown $13^{\text {th }}$ September to 26 th November.

\begin{tabular}{|c|c|c|c|c|c|c|}
\hline \multirow{2}{*}{ Populations } & & \multicolumn{3}{|c|}{ Seedling derived (s) clones } & \multicolumn{2}{|c|}{ Original $(c)$ clones } \\
\hline & & $3^{s}$ & $17 s$ & $673^{8 s}$ & $3 c$ & $17 c$ \\
\hline Genotype $1-$ High & . $\quad$. & $\begin{array}{l}22 \\
66\end{array}$ & $\begin{array}{l}32 \\
57\end{array}$ & $\begin{array}{l}23 \\
59\end{array}$ & $\begin{array}{l}33 \\
55\end{array}$ & $\begin{array}{l}25 \\
49\end{array}$ \\
\hline Low & . & $\begin{array}{l}15 \\
33\end{array}$ & $\begin{array}{l}30 \\
46\end{array}$ & $\begin{array}{l}16 \\
69\end{array}$ & $\begin{array}{l}25 \\
57\end{array}$ & $\begin{array}{l}29 \\
51\end{array}$ \\
\hline Genotype 2-High & . $\quad$. & $\begin{array}{l}16 \\
51\end{array}$ & $\begin{array}{l}29 \\
70\end{array}$ & $\begin{array}{l}24 \\
6 i\end{array}$ & $\begin{array}{l}33 \\
64\end{array}$ & $\begin{array}{l}38 \\
49\end{array}$ \\
\hline Low & . $\quad$. & $\begin{array}{l}14 \\
3^{6}\end{array}$ & $\begin{array}{l}29 \\
68\end{array}$ & $\begin{array}{l}24 \\
54\end{array}$ & $\begin{array}{l}33 \\
61\end{array}$ & $\begin{array}{l}3^{8} \\
3^{8}\end{array}$ \\
\hline
\end{tabular}

Analysis of variance

(using measurements from daughter ramets $=$ assessment phase)

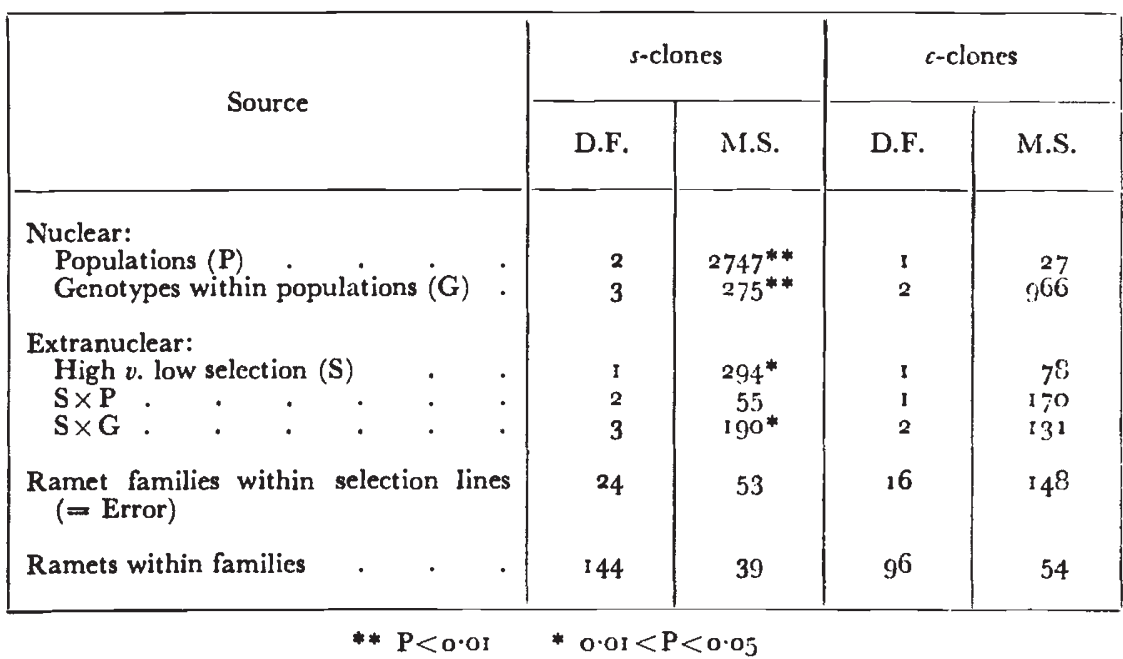

assuming a two-tail distribution and so, as discussed earlier, tend to be conservative for items measuring selection effects.

Taking first the $s$-clones, main-effects items for populations and for genotypes within populations are significant, indicating differences of a nuclear origin both between and within populations. The mean 
square measuring the overall effect of selection is significant at the 5 per cent. level and is of the same order as that for genotypes within populations. Thus somatic selection within clones has produced differences at least as great as those attributable to nuclear differences in the population. This, indeed, is apparent in the graphs for $3 \mathrm{~s}$ and $17 s$ in fig. 1 . The interaction of selective response and genotypes within populations is significant and of the same order as that for overall selection, but unlike the previous analysis there is no significant interaction at the population level.

At this stage, which it must be remembered follows a cycle of relaxed selection, there is therefore again evidence of genotype

TABLE 3

Analysis of covariation between parent and daughter ramets

(using mean values of table 2 and excluding population $673^{8}$ )

\begin{tabular}{|c|c|c|c|c|c|}
\hline Source & D.F. & $\begin{array}{c}\text { M.S. } \\
\text { (parents) }\end{array}$ & M.C.P. & $\begin{array}{c}\text { M.S. } \\
\text { (daughters) }\end{array}$ & $r$ \\
\hline Seedling-derived (s) clones: & & & & & \\
Between clones (nuclear) \\
Within clones (extranuclear) & 3 & 238 & 120 & 124 & $0 \cdot 70$ \\
Original (c) clones: & 4 & 178 & 35 & 7 & $0 \cdot 98^{* * *}$ \\
Between clones (nuclear) & & & & & \\
Within clones (extranuclear) & 3 & 129 & -22 & 43 & $-0 \cdot 29$ \\
\hline
\end{tabular}

$* * * \mathrm{P}<0.001$

dependency in response to selection but none to indicate that the origin of the genotypes is important.

Turning to the analysis of $c$-clonal variation, it will be seen that none of the items is significantly greater than the basic error. The M.S. for ramets within families is significantly lower than that for family means, but as already discussed this may be a reflection of the inadequate experimental design. The analyses thus confirm the previous results in being unable to detect any responses to selection. It is perhaps surprising that no differences of a nuclear kind are found. This could be due to a past selection for similar genotypes or indeed to selection for uniformity in expression through the medium of the extranuclear component of variation which has been demonstrated in the $s$-clones. However, there is an instability in these old-established $c$-clones which might well transcend other effects. It is apparent in the graphs of fig. I as well as indicated by the relatively large mean square for ramet families within lines, compared with the same item for the s-clones. This variation, which apparently cannot be seized upon by selection, may well not be without significance and may be related to the age of the clones. The possible significance of this is discussed later. 
Table 3 sets out a simplified form of analysis of covariation between parent and daughter ramets of the assessment phase, using the mean values of table 2 and excluding population $6738 \mathrm{~s}$. In the $c$-clones the results of the previous analyses are borne out by the non-significant parent/daughter correlations. In the $s$-clones it is of interest to note that there is a diminution of the within-clonal variation relative to that between clones from the parent stage to the daughter stage, which is reflected in the increased variance ratios of the two mean squares measuring these effects $(=1 \cdot 3$ for parents and $17 \cdot 7$ for daughters). This suggests that some of the differences built up by somatic selection within clones has been dissipated during the cycle of relaxed selection. Nevertheless the parent/daughter correlation within clones is high and significant and is in fact greater, although not significantly so, than that obtained for the between-clonal (or nuclear) fraction of the variation. The important, perhaps even the dominating role of the extranuclear component in controlling variation in the rate of asexual reproduction, is thus again emphasised.

\section{DISCUSSION}

First and foremost the experiment has shown that in certain clones of perennial ryegrass the rate of tillering can be significantly influenced by somatic selection. Libby and Jund ( 1962 ) have demonstrated that variation in clones of Mimulus guttatus may arise as the result of local differences in the micro-environment or as a result of inequalities between propagules. In our experiment, at each cloning stage, the selection lines were allocated at random to positions in the glasshouse and care was taken to try and ensure that tillers were of a comparable developmental stage; thus differences due to the micro-environment or differential stages of development would not likely be perpetuated over four selection cycles. Rather must we look for more permanent changes in the internal metabolism, controlled at the cellular level and transmitted over tiller generations. Within clones the genotype will of course be constant barring mutation which must be adjudged too infrequent to affect the course of these experiments. Therefore the differences between selection lines can most reasonably be ascribed to extranuclear or cytoplasmic inequalities arising during asexual propagation, which have been seized upon and magnified by selection. The differential selection lines may thus be expected to differ in plasmagene content (the plasmon) and may be termed plasmatypes following the terminology of Michaelis and Jones cited by Jinks ( 1964$)$. This terminology is followed in subsequent discussion.

The nature of these extranuclear differences is not always easy to interpret. Thus in fungi it has been found that differences can occur as the result of degeneration of the cytoplasm during ageing, whilst in other instances they may have adaptive significance (Jinks, 
1957, 1959 $a$ and $b$ ). Indeed, as discussed by Mather (1954) and Jinks (1956, 1959b), the process of ageing may itself be a result of degeneration or imbalance in those plasmic constituents which are also the raw material for adaptive selection, and as a consequence the two processes are difficult to divorce.

The question is thus posed as to whether the differences produced here have any adaptive significance, or whether the high lines merely represent selection against degenerative changes which are manifested as a slower growth rate in the low lines. Certainly degenerative changes during vegetative reproduction, in particular those affecting sexual processes, have been observed in perennial ryegrass and these have been associated with clonal age (Beddows, Breese and Lewis, I962).

At this point it is germane to examine more closely the method of selection employed in the experiment. For each line and at each stage, selection was based on the tiller numbers achieved by a set of three daughter ramets after an interval of time. The selected ramet was then broken down into tillers and three vigorous tillers of uniform appearance and development were taken to generate ramets of the next selection cycle. This, therefore, involved dual and not necessarily reinforcing selections. In the first case an adjustment of the rate of tillering was sought by selection among daughter ramets; in the second case the more gross effects of plasmic deterioration affecting tiller development were eliminated by selecting uniformly vigorous tillers within selected ramets. The selection thus aimed at adjustment in the rate of tillering within the limits set by retaining metabolically functional lines, and so sought to exploit that part of the extranuclear variation which might be reasonably expected to have adaptive significance under sward conditions.

Evidence that the aim of the selection has been achieved derives from the contrasting reaction of the $s$-clones and $c$-clones of populations 3 and 17 . The chief difference between these groups was in clonal age, the $c$-clones being derived by vegetative propagation from the original swards, while the $s$-clones derived from seedlings produced by a single sexual cycle in the same populations. Selection produced no response in the c-clones whereas, by contrast, response was immediate and marked in a proportion of the $s$-clones. Now if selection merely picked out plasmic differences which were degenerative in origin, it should be at least_as effective in the $c$-clones as in the $s$-clones, or even more so, since the $c$-clones might be expected to show instabilities associated with their greater age. Indeed the experiment itself provides evidence of a greater spontaneous or segregational variation within the $c$-clones and a high proportion of this can be reasonably assumed to be age-conditioned degenerative effects. Since no response was obtained in these clones it argues that such effects are largely discounted by the method of selection used, which might therefore be presumed to produce only changes which are potentially adaptive. 
Accepting this, however, the obvious corollary is that adaptive changes us e nnly found in the younger, seedling-derived clones and the significance of this is discussed below.

Having shown that extranuclear variation of an adaptive nature exists, we can now consider its role in sward dynamics. It cannot be doubted that the rate of tillering, measuring as it does the rate of asexual reproduction, must bear on competitive ability and finally on persistency in natural populations. In long established swards, where management has kept sexual regeneration to a minimum, selection for the appropriate rate of asexual reproduction must have been at a premium, and it may be expected that vegetative derivatives would be relatively fixed, or homoplasmic, for that part of the extranuclear system governing variation in this character. It is thus significant that selection could produce no responses in the vegetatively derived $c$-clones of populations 3 and 17 , and it may be inferred that continued asexual propagation, much of it under sward conditions, has resulted in a stable homoplasmic state. But the potentially adaptive differences produced in the seedling clones make it evident that a single cycle of sexual reproduction restores an equilibrium state in the system; a state which can be likened to a heteroplasmon. In this respect the situation is analagous to that found in fungi (Mather and Jinks, 1958), where cytoplasmic balance can be profoundly modified during asexual propagation but restored by a sexual phase.

A striking feature of the experiment was that such marked differences were obtained in certain of the s-clones over a relatively short period of time by an extremely low selection pressure. This implies a lability in heteroplasmic clones which argues that the plasmon may have considerable significance in sward dynamics. Thus the experiment shows that in certain populations plasmatypic differences could exceed those between genotypes, so that phenotypic uniformity in a sward need not bespeak a genotypic similarity. In this respect Breese and Charles (1962) find a high degree of phenotypic uniformity within populations of perennial ryegrass vegetatively derived from old-established pastures, which is unlikely to be due to sampling identical genotypes. (Populations 3 and 17 , used in the present experiment, formed part of this study.)

The experiment also showed clearly that even at the potentially labile post-seedling phase, the ability to respond to somatic selection was under genotypic control. The graphs of fig. 2 suggest that in this respect the origin of the genotypes was important, and indicate that the ability to respond was predisposed by a history of adaptation to asexual propagation previous to the sexual cycle. Admittedly the number of genotypes sampled in each population was so low that these relationships should not be overemphasised. Nevertheless attention is thereby drawn to the importance of nuclear/plasmic complexes under sward conditions, and also poses the question as to how, and to what extent, plasmic properties are transmitted through

2 A 2 
sexual generations. In the present experiment seed was derived from intra-population crosses, but unpublished data from inter-population crosses in adjunct studies reveal a high frequency of reciprocal differences in mature plant characteristics which may well have significance in this context.

The results generally suggest that the differentiation and stabilisation of ryegrass populations may be a more complex genetical process than has hitherto been considered. During the establishment phase of a newly seeded sward, Charles ( $196 \mathrm{I}$ ) has shown a striking reduction in the number of individual plants. At this initial stage, therefore, it may be assumed that selection is entirely at the genotypic level. But if regeneration by sexual means is prohibited, continued survival will depend on asexual propagation and selective adjustment will finally come to depend on variation at the plasmatypic level. For mature sward characteristics, therefore, expression may well depend on plasmon lability and nuclear selection may be relegated to picking out genotypes which predispose this lability.

This process poses many problems, particularly of the maintenance of plasmon lability. Thus, although a cycle of relaxed selection suggested that initial responses were reversible, results from the old established clones indicated that these had become homoplasmic and hence non-adaptable. However this initial and tentative experiment must inevitably pose more questions than it can seek to answer. Future work seeks to investigate more specifically the effects of clonal age, the effect on the sexual processes of selection at the plasmon level, and particularly to what extent plasmon lability can confer an adaptive plasticity to the plant under differing edaphic and management conditions.

\section{SUMMARY}

An experiment has shown that the rate of tillering (= asexual reproduction) in perennial ryegrass can be significantly altered by selection within clones. The response to this somatic selection depended on the age of the clone such that response was obtained in young clones raised from seedlings but not in clones with a long history of uninterrupted asexual propagation. The response was also dependent on the genotype of the clone and there was some evidence to suggest that the somatic lability of genotypes was predisposed by a history of adaptation to asexual propagation previous to the sexual cycle. The responses could be attributed to changes in the plasmon and were inferred to be potentially adaptive. The significance of these results in relation to sward dynamics is discussed.

\section{REFERENCES}

BEDDOWS, A. R., BREESE, E. L., AND LEWIS, B. I962. The genetic assessment of heterozygous breeding material by means of a diallel cross. Heredity, 17 , 501-512. 
BReEse, E. L., AND Gharles, A. H. 1962. Population studies in ryegrass. Rep. Welsh Pl. Breed. Sta., 1961, 30-34.

Charles, A. H. 1961. Differential survival of cultivars of Lolium, Dactylis and Phleum. 7. Brit. Grassl. Soc., 16, 69-75.

JnNks, J. L. 1956. Naturally occurring cytoplasmic changes in fungi. Compt. rend. Lab. Carlsberg. s. Physiol., 26, 183-203.

Junks, J. L. 1957. Selection for cytoplasmic differences. Proc. Roy. Soc. B., I46, 527.540.

JiNKs, J. L. 1959a. Selection for adaptability to new environments in Aspergillus glaucus. F. gen. Microbiol., 20, 223-236.

JiNks, J. L. 1959b. Lethal suppressive cytoplasms in aged clones of Aspergillus glaucus. F. gen. Microbiol., 21, 397-409.

JiNks, J. L. 1964. Extrachromosomal inheritance. Prentice-Hall, Inc., New Jersey. $176 \mathrm{pp}$.

LANGRR, R. H. M. 1956. Growth and nutrition of timothy (Phleum pratense). (i). The Life history of individual tillers. Ann. appl. Biol., 44, 166-187.

LIBBY, W. J., AND JUND, E. 1962. Variance associated with cloning. Heredity, I7, 533.540.

MATHER, K. 1954. Age of parent and character of offspring. Nature, 174, 304.

MATHER, K., AND JINks, J. L. 1958. Cytoplasm in sexual reproduction. Nature, I82, I 188-1 I 90 . 